\title{
Vision-Based Control of Robot Motion
}

\author{
Seth Hutchinson \\ Dept. of Electrical and Computer Engineering - The Beckman Institute \\ University of Illinois, USA \\ seth@uiuc.edu \\ http://www-cvr.ai.uiuc.edu/ seth/
}

\begin{abstract}
Visual servo control is now a mature method for controlling robots using real-time vision feedback. It can be considered as the fusion of computer vision, robotics and control, and it has been a distinct field since the 1990's, though the earliest work dates back to the 1980's. Over this period several major, and well understood, approaches have evolved and have been demonstrated in many laboratories around the world. Many visual servo schemes can be classified as either position-based or image-based, depending on whether camera pose or image features are used in the control law. This lecture will review both position-based and image-based methods for visual servo control, presenting the basic derivations and concepts, and describing a few of the performance problems faced by each. Following this, a few recent and more advanced methods will be described. These approaches essentially partition the control system either along spatial or temporal dimensions. The former are commonly referred to as hybrid or partitioned control systems, while the latter are typically referred to as switched systems.
\end{abstract}

